\title{
UNDERDETERMINED BLIND SOURCE SEPARATION OF NON-DISJOINT NONSTATIONARY SOURCES IN THE TIME-FREQUENCY DOMAIN
}

\author{
N. Linh-Trung
}

\author{
CNES, 18 avenue Edouard Belin \\ 31401, Toulouse Cedex 9, France \\ linh-trung.nguyen@cnes.fr
}

\author{
A. Aïssa-El-Bey, K. Abed-Meraim \\ ENST-Paris, 46 rue Barrault \\ 75634, Paris Cedex 13, France \\ \{elbey, abed\}@tsi.enst.fr
}

\section{A. Belouchrani}

\author{
ENP, B.P 182 El-Harrach, \\ Algiers 16200, Algeria \\ adel.belouchrani@enp.edu.dz
}

\begin{abstract}
This paper considers the blind separation of nonstationary sources in the underdetermined case, in which we have more sources than sensors. A recently proposed algorithm applied time-frequency distributions (TFDs) to this problem and gave good separation performances in the case where sources were disjoint in the time-frequency (TF) plane. However, in the non-disjoint case, the method simply relied on the interpolation at the intersection TF points implicitly performed by a TF synthesis algorithm, instead of directly treating these points. In this paper, we propose a new algorithm that combines the abovementioned method with subspace projection in order to explicitly treat non-disjoint sources. Another contribution of this paper is the estimation of the mixing matrix in the underdetermined case.
\end{abstract}

\section{INTRODUCTION}

Blind source separation (BSS) considers the recovery of unobserved original sources from several mixtures which are observed at the output of a set of sensors. Each mixture contains a combination of the sources that is resulted from the mixing medium between the sources and the sensors. The term "blind" indicates that no a priori knowledge of both the sources and the medium structure is available. To compensate for this lack of information, the sources are usually assumed to be statistically independent. BSS has application in different areas, such as communication, speech and image processing and biomedical engineering [1].

A challenging problem of BSS arises when there are more sources than sensors; this is now referred to as underdetermined blind sources separation (UBSS). A TFbased UBSS algorithm has been recently proposed in [2] to successfully separate nonstationary sources using TFDs. This algorithm gave good performances when the sources were disjoint in the TF plane. It also provided the separation of TF quasi-disjoint sources, that is the sources were allowed to have a small degree of overlapping in the TF plane.

However, the intersection TF points were not directly treated. In particular, a point at the intersection of two sources is clustered "by chance" to belong to one of the sources. As a result, the source that has picked up this point now has some information of the other source while the later lost some information of its own. The lost information can be fortunately recovered to some extent by the interpolation at the intersection point using TF synthesis. However, for the other source, there is an interference at this point, hence the separation performance may degrade if no treatment is provided for this. The more the number of intersection points not to be treated, the worse the interpolation result and the interference would become, hence the worse the final separating performance.

In this paper, we propose another algorithm that combines the above TF-UBSS algorithm with subspace projection, offering an explicit treatment of the intersection points. The main assumption used in this algorithm is that the number of sources simultaneously present at any intersection point must be smaller than the total number of sensors.

\section{DATA MODEL AND ASSUMPTIONS}

Let $N$-dimensional vector $\mathbf{s}(t)=\left[s_{1}(t), \ldots, s_{N}(t)\right]^{T}$ represent $N$ nonstationary source signals. The source signals are transmitted through a medium so that an array of $M$ linear sensors picks up a set of mixed signals represented by an $M$-dimensional vector $\mathbf{x}(t)=\left[x_{1}(t), \ldots, x_{M}(t)\right]^{T}$. Consider the instantaneous mixing model, as given by:

$$
\mathbf{x}(t)=\mathbf{A} \mathbf{s}(t)+\mathbf{w}(t)
$$

where $\mathbf{A}=\left[\mathbf{a}_{1}, \ldots, \mathbf{a}_{N}\right]$ is the mixing matrix and $\mathbf{w}(t)=$ $\left[w_{1}(t), \ldots, w_{M}(t)\right]^{T}$ is the observation noise vector. The goal of BSS is to recover $\mathbf{s}(t)$ from $\mathbf{x}(t)$. When $M<N$, the problem becomes UBSS. In this case, we assume that any $M$ column vectors of $\mathbf{A}$ are linearly independent.

In [2], sources are assumed to be disjoint in the TF plane; that is, for any pair of two sources $s_{1}(t)$ and $s_{2}(t)$ whose TF signatures are denoted by $\Omega_{1}$ and $\Omega_{2}$ respectively, we have $\Omega_{1} \cap \Omega_{2} \neq \emptyset$. The notion of TF disjoint is illustrated in Fig.1-a.

In this paper, we relax the above assumption by allowing the sources to be generally non-disjoint, but limiting the degree of disjoint to the following two conditions. First, there are at most $(M-1)$ sources present at any TF point. This allows us to apply the subspace projection approach for the source TFDs estimation, to be shown in Section 4. Second, for each source signal, there exists a 


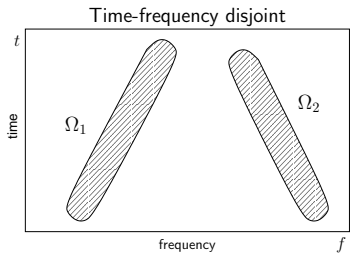

(a)

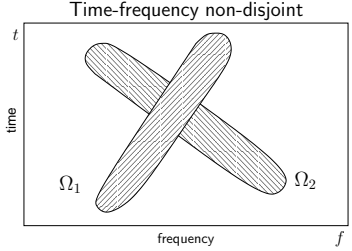

(b)
Fig. 1. (a)-TF disjoint, (b) TF non-disjoint

region in the TF plane where the source exists alone; in other words, the energy of the other sources are negligible at the TF points within the considered region. This is needed for the estimation of the mixture matrix, also to be shown in Section 4. In the case the latter condition is not satisfied, one can always estimate the mixing matrix using algebraic [6] or other approaches. The TF non-disjoint is illustrated in Fig.1-b.

\section{SOURCE SEPARATION USING TFD}

In this section, we briefly review the TF-UBSS method proposed in [2]. This method uses the spatial TFD (STFD) that is defined in [3] by the following:

$$
\begin{aligned}
\mathbf{D}_{\mathbf{x x}}(t, f)= & \sum_{l=-\infty}^{+\infty} \sum_{k=-\infty}^{+\infty} \phi(k, l) . \\
& \mathbf{x}(t+k+l) \mathbf{x}^{H}(t+k-l) e^{-j 4 \pi f l},
\end{aligned}
$$

where $\phi(k, l)$ is the TFD time-lag kernel and the superscript $\left.{ }^{H}\right)$ denotes the complex conjugate transpose operator. The matrix $\mathbf{D}_{\mathbf{x x}}(t, f)$ varies with respect to $t$ and $f$. When evaluated at a particular TF point, its $(i, j)$-element has the value of the cross-TFD of $x_{i}(t)$ and $x_{j}(t)$ at this point.

Applying (2) to the linear data model in (1), while assuming that the additive noise is not present, leads to the following expression:

$$
\mathbf{D}_{\mathbf{x} \mathbf{x}}(t, f)=\mathbf{A D}_{\mathbf{s}}(t, f) \mathbf{A}^{H},
$$

where $\mathbf{D}_{\mathbf{x x}}(t, f)$ is now the mixture STFD matrix and $\mathbf{D}_{\mathbf{s s}}(t, f)$ is the source STFD matrix. After computing the above mixture STFD matrix (we did use here the WignerVille distribution (WVD) or the Modified-WVD (MWVD) [4]) and using noise thresholding, one proceeds then to the selection of auto-source TF points; these are the points at each of which only one source is active. This selection is done following the procedure in [7].

The structure of the mixture STFD is as such: at an auto-source TF point, there is only one value not equal to zero among its diagonal elements. Therefore, if all sources are disjoint in the TF plane then, for all TF points belong to the TF signature $\Omega_{i}$ of a source $s_{i}(t)$, we have

$$
\mathbf{D}_{\mathbf{x x}}(t, f)=D_{s_{i} s_{i}}(t, f) \mathbf{a}_{i} \mathbf{a}_{i}^{H},
$$

where $D_{s_{i} s_{i}}(t, f)$ is the TFD of $s_{i}(t)$. In [6], $\mathbf{a}_{i}$ represents the principal eigenvector of $\mathbf{D}_{\mathbf{x x}}(t, f)$ and $D_{s_{i} s_{i}}(t, f)$ is (up to a constant) the corresponding eigenvalue, or equivalently the trace value, of $\mathbf{D}_{\mathbf{x x}}(t, f)$. Therefore, all the auto-source points that belong to one particular source must have the same spatial direction. In other words, if we cluster these points into classes corresponding to different spatial directions, then these classes represent the individual source signals.

Given the class $C_{i}$ representing the source $s_{i}(t)$, the TFD estimate of $s_{i}(t)$ is then computed (up to a constant factor) by:

$$
\begin{aligned}
& \hat{D}_{s_{i} s_{i}}(t, f)= \\
& =\left\{\begin{array}{ll}
\operatorname{trace}\left\{\mathbf{D}_{\mathbf{x x}}^{\mathrm{wvd}}\left(t_{a}, f_{a}\right)\right\}, & \forall\left(t_{a}, f_{a}\right) \in C_{i} \\
0, & \text { otherwise }
\end{array} .\right.
\end{aligned}
$$

Having obtained the source TFD estimates $\hat{D}_{s_{i} s_{i}}$, we then use an adequate source synthesis procedure to estimate the source waveform $s_{i}(t)$. The recovery of the source waveform from its TFD is made possible thanks to the following inversion property of the WVD [4]

$$
x(t)=\frac{1}{x^{*}(0)} \int_{-\infty}^{\infty} \rho_{x}^{\mathrm{wvd}}\left(\frac{t}{2}, f\right) e^{j 2 \pi f t} d f,
$$

which implies that the signal can be reconstructed to within a complex exponential constant $e^{j \alpha}=x^{*}(0) /|x(0)|$ given that $|x(0)| \neq 0$. The method in [2] uses the synthesis algorithm that is proposed in [5]. This algorithm recovers the source waveform from its WVD estimates.

In brief, the TF-UBSS algorithm in [2] can be summarized in the following four steps:

\section{Tab.1: TF-UBSS algorithm}

S1: STFD computation and noise thresholding

S2: Auto-source TF point selection

S3: Clustering and source TFD estimation

S4: Source signal synthesis

\section{TF-UBSS USING SUBSPACE PROJECTION}

As explained in the introduction, the TF-UBSS method reviewed in Section 3 did not treat the intersection points properly in the case where the sources are non-disjoined in the TF plane. We propose here to use an appropriate subspace projection to estimate the TFDs of the individual sources from the selected auto-source points, under the assumption that there are at most $(M-1)$ sources simultaneously present at any given point.

In particular, consider an auto-term point $\left(t_{a}, f_{a}\right)$ at which sources $s_{i_{1}}, \ldots, s_{i_{I}}$ contribute $(I<M)$ and define $\widetilde{\mathbf{s}}=\left[s_{i_{1}}, \ldots, s_{i_{I}}\right]^{T}$ and $\widetilde{\mathbf{A}}=\left[\mathbf{a}_{i_{1}}, \ldots, \mathbf{a}_{i_{I}}\right]^{T}$. We have then

$$
\mathbf{D}_{\mathbf{x x}}\left(t_{a}, f_{a}\right)=\widetilde{\mathbf{A}} \mathbf{D}_{\widetilde{\mathbf{s}}}\left(t_{a}, f_{a}\right) \widetilde{\mathbf{A}}^{H}
$$

and consequently (assumed that $\mathbf{D}_{\widetilde{\mathbf{s}}}\left(t_{a}, f_{a}\right)$ is of full rank)

$$
\operatorname{Range}\left(\mathbf{D}_{\mathbf{x x}}\left(t_{a}, f_{a}\right)\right)=\operatorname{Range}(\widetilde{\mathbf{A}}) .
$$


Let us now assume that $\mathrm{A}$ is known (or already estimated), and proceed with the estimation of the source TFDs. One can obtain information about contributing sources at this point by observing that:

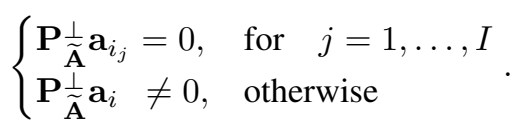

Above, $\mathbf{P}_{\widetilde{\mathbf{A}}}^{\perp}$ represents the orthogonal projection matrix onto the noise subspace of $\mathbf{D}_{\mathbf{x x}}\left(t_{a}, f_{a}\right)$ and can be computed by

$$
\mathbf{P}_{\widetilde{\mathbf{A}}}^{\perp}=\mathbf{I}-\mathbf{V} \mathbf{V}^{H}
$$

where $\mathbf{V}$ is the matrix of the $I$ principal singular eigenvectors of $\mathbf{D}_{\mathbf{x x}}\left(t_{a}, f_{a}\right)$. In practice, to take into account estimation noise, one detects the $I$ column vectors of $\widetilde{\mathbf{A}}$ as the vectors of $\mathbf{A}$ corresponding to the $I$ smallest values of the set $\left\{\left\|\mathbf{P} \underset{\widetilde{\mathbf{A}}}{\perp} \mathbf{a}_{i}\right\|\right\}$ where $i=1, \ldots, N$. Once $\widetilde{\mathbf{A}}$ is obtained, we estimate the TFDs of $I$ sources at point $\left(t_{a}, f_{a}\right)$ as the diagonal entries of:

$$
\widetilde{\mathbf{A}}^{\#} \mathbf{D}_{\mathbf{x x}}\left(t_{a}, f_{a}\right)\left(\widetilde{\mathbf{A}}^{\#}\right)^{H} \approx \mathbf{D}_{\widetilde{\mathbf{s}}}\left(t_{a}, f_{a}\right)
$$

In the estimation of source TFDs above, we have assumed that $\mathbf{A}$ had been known a priori or already been estimated. Along with other existing methods, we propose here a method to estimate $\mathbf{A}$ by using the assumption that for each source signal $s_{i}$ there exists a TF region $\mathbf{R}_{i}$ where

$$
\mathbf{D}_{\mathbf{x x}}(t, f)=D_{s_{i} s_{i}}(t, f) \mathbf{a}_{i} \mathbf{a}_{i}^{H}, \quad \forall(t, f) \in \mathbf{R}_{i} .
$$

Based on that observation we estimate $\mathbf{A}$ as follows.

- First, detect the TF points belonging to the region $\mathcal{R}=\bigcup_{i=1}^{N} \mathbf{R}_{i}$ using the test criterion

$$
(t, f) \in \mathcal{R} \quad \text { iff } \quad\left|\frac{\lambda_{\max }\left\{\mathbf{D}_{\mathbf{x x}}(t, f)\right\}}{\operatorname{trace}\left\{\mathbf{D}_{\mathbf{x x}}(t, f)\right\}}-1\right|<\epsilon,
$$

where $\epsilon$ is a small threshold value (typically $\epsilon \leq$ $0.1)$ and $\lambda_{\max }\left\{\mathbf{D}_{\mathbf{x x}}(t, f)\right\}$ denotes the maximum eigenvalue of $\mathbf{D}_{\mathbf{x x}}(t, f)$.

- Then, for each point $(t, f)$ in $\mathcal{R}$, estimate the vector $\widehat{\mathbf{a}}(t, f)$ as the principal eigenvector of $\mathbf{D}_{\mathbf{x x}}(t, f)$.

- Finally, cluster the set of vectors $\{\widehat{\mathbf{a}}(t, f)\}$, where $(t, f) \in \mathcal{R}$, into $N$ classes $^{1}$ using any vector clustering technique existing in the literature [8]. In this paper, we have used the algorithm k-means. The column vectors of $\mathbf{A}$ are estimated as the $N$ centroids of the $N$ clusters.

Table 2 presents a summary of the subspace projection based TF-UBSS algorithm.

\footnotetext{
${ }^{1}$ There exist techniques that perform both the clustering and the estimation of the number of classes. For simplicity, we assumed here the number of sources known.
}

\section{Tab.2: subspace-based TF-UBSS algorithm}

S1: STFD computation and noise thresholding

S2: Auto-source TF point selection

S3: Selection of rank-1 STFD matrices and computation of their respective principal eigenvectors S4: Clustering of the previous set of vectors and estimation of column vectors of $\mathbf{A}$ as the centroid points

S5: For all auto-source TF point, perform the subspace based TFD estimation

S6: Source signal synthesis

\section{SIMULATION RESULTS}

In the simulation, we use a uniform linear array of $M=3$ sensors having half wavelength spacing. It receives signals from $N=4$ independent linear frequency-modulated sources, each has 256 samples, in the presence of additive Gaussian noise where the $\mathrm{SNR}=20 \mathrm{~dB}$.

The effect of cross-terms on the WVD representation of one the mixture (as shown in Fig.2-(a)) was reduced by using MWVD (as shown in Fig.2-(b)). Fig.2-(d) displays the auto-source TF points using MWVD. Fig.2-(c) re-displays these points but with the TFD values extracted from the WVD in Fig.2-(a); these TFD values will be used for TFD estimation.

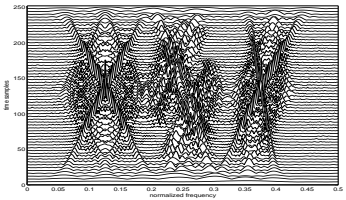

(a) mixture (WVD)

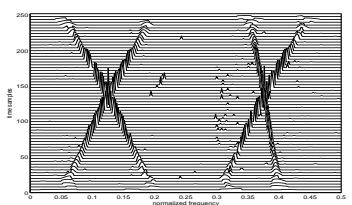

(c) source TF points (WVD)

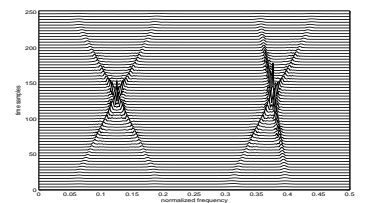

(b) mixture (MWVD)

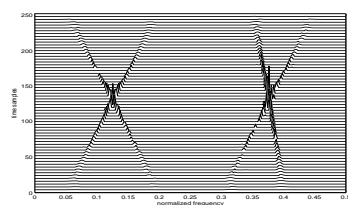

(d) source TF points (MWVD)
Fig. 2. TFD choices and auto-source selection.

We compare the algorithm proposed in [2] (now referred to as cluster-based TF-UBSS) with the algorithm proposed in this paper, now referred to as subspace based TF-UBSS. Note that Fig.3-(b,e,h,k) show the estimated source TFDs using the cluster-based algorithm, whereas Fig.3-(c,f,i,l) are those obtained by the subspace based algorithm.

From Fig.3-(b,e), we can see that the intersection points between source $s_{1}(t)$ and source $s_{2}(t)$ were picked up by source $s_{2}(t)$ by the cluster-based algorithm. On the other hand, using the subspace-based algorithm, the intersection points have been redistributed to the two sources (Fig.3$(\mathrm{c}, \mathrm{f}))$.

In Fig.4, we provide a statistical evaluation of the performance gain we observed in the previous experiment. 
For that, we run $N_{r}=500$ Monte Carlo trials with the same sources but random noise realization at each trial. The quality of source extraction is measured by the following normalize MSE

$$
\mathrm{MSE}=\frac{1}{N_{r}} \sum_{r=1}^{N_{r}} \frac{\left\|\widehat{\mathbf{s}}_{r}-\mathbf{s}\right\|^{2}}{\|\mathbf{s}\|^{2}},
$$

where $\widehat{\mathbf{s}}_{r}$ represents the estimate of the sources at the $r$-th trial. Note that for comparison we remove the scalar and permutation indeterminacies. Even though the considered sources are almost disjoint in the TF domain, we observe an improvement of the separation method thanks to subspace projection. We expect this improvement to be more significant if the sources have larger intersection region in TF plane.

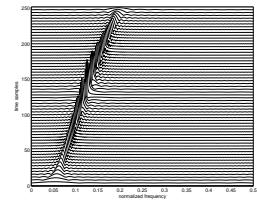

(a) $W_{s_{1}}(t, f)$

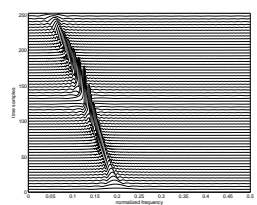

(d) $W_{s_{2}}(t, f)$

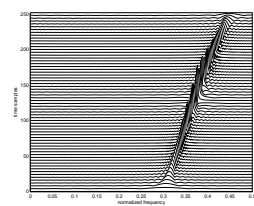

(g) $W_{s_{3}}(t, f)$

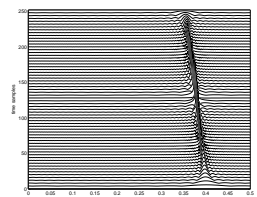

(j) $W_{s_{1}}(t, f)$

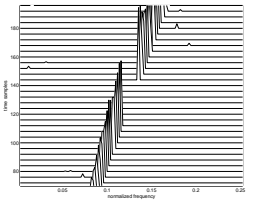

(b) $\hat{W}_{s_{1}}$ (cluster)

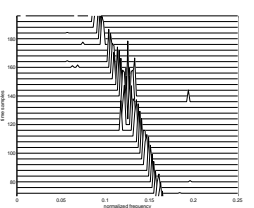

(e) $\hat{W}_{s_{2}}$ (cluster)

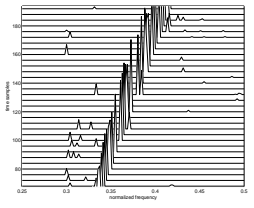

(h) $\hat{W}_{s_{3}}$ (cluster)

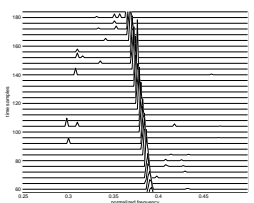

(k) $\hat{W}_{s_{4}}$ (cluster)

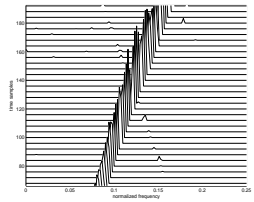

(c) $\hat{W}_{s_{1}}$ (subspace)

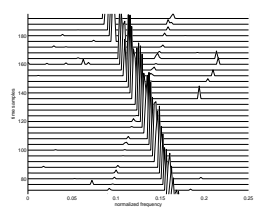

(f) $\hat{W}_{s_{2}}$ (subspace)

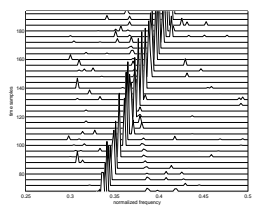

(i) $\hat{W}_{s_{3}}$ (subspace)

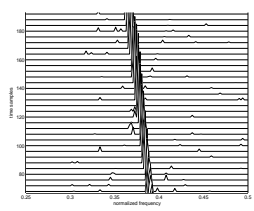

(1) $\hat{W}_{s_{4}}$ (subspace)
Fig. 3. TFD estimation.

\section{CONCLUSION}

This paper introduces a new approach for blind separation of non-disjoint and nonstationary sources using TFDs. The proposed method can separate more sources than sensors and provides, in the case of non-disjoint sources, a better separation quality than the method proposed in [2]. This method is based on a vector clustering procedure that estimates the mixing matrix $\mathbf{A}$, and subspace projection to separate the sources at the intersection points in the TF plane.

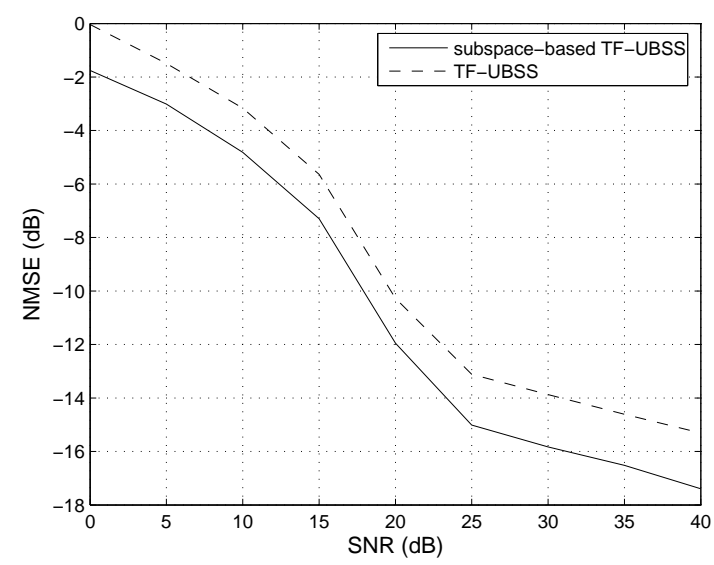

Fig. 4. MSE versus SNR.

\section{REFERENCES}

[1] A. K. Nandi, Ed., Blind Estimation Using HigherOrder Statistics, Boston: Kluwer Academic, 1999.

[2] N. Linh-Trung, A. Belouchrani, K. Abed-Meraim and B. Boashash, "Separating more sources than sensors using time-frequency distributions," EURASIP J. of Applied Sig. Proc., vol. 2005, no. 16.

[3] A. Belouchrani and M. G. Amin, "Blind source separation based on time-frequency signal representations," IEEE Trans. on Sig. Proc., vol. 46, no. 11, pp. 2888-2897, Nov. 1998.

[4] B. Boashash, Ed., "Time Frequency Signal Analysis and Processing: Method and Applications," Elsevier, Oxford, 2003.

[5] G. F. Boudreaux-Bartels and T. W. Marks, "Timevarying filtering and signal estimation using Wigner distributions," IEEE Trans. on Acous., Speech, and Sig. Proc., vol. 34, pp. 422-430, Mar. 1986.

[6] L. De Lathauwer, B. Moor, J. Vandewalle, "ICA techniques for more sources than sensors," Higher-order statistic Proc. of the IEEE Sig. Proc. Workshop, pp. 116-120, 1999.

[7] A. Belouchrani, K. Abed-Meraim, M.G. Amin, A.M. Zoubir, "Blind separation of nonstationary sources," Signal Processing Letters, IEEE, vol. 11, pp. 605-608, 2004.

[8] I.E. Frank and R. Todeschini, "The data analysis handbook", Elsevier, Sci. Pub. Co, 1994. 